Jarauta Borrasca, B., Medina Moya, J. L. y Mentado Labao, T. (2016). La transformación del saber en la enseñanza universitaria. Una aproximación desde el estudio del CDC. Revista de Investigación Educativa, 34(2), 471-485. DOI: http://dx.doi.org/10.6018/rie.34.2.221711

\title{
La transformación del saber en la enseñanza universitaria. Una aproximación desde el estudio del CDC*
}

\author{
Transforming knowledge in university education. \\ A PCK studies approach
}

\author{
Beatriz Jarauta Borrasca, José Luis Medina Moya y Trinidad Mentado Labao \\ Departamento de Didáctica y Organización Educativa. Facultad de Educación. Universidad de Barcelona. España.
}

\begin{abstract}
Resumen
Este artículo tiene como objetivo mostrar el proceso seguido y los resultados obtenidos en una investigación dirigida a conocer cómo se manifiesta el Conocimiento Didáctico del Contenido (CDC) en la enseñanza universitaria, y en concreto en las transformaciones que el profesorado realiza sobre sus materias de enseñanza bajo el objeto de hacerlas más comprensibles a los estudiantes. La investigación, desarrollada a través de un estudio de casos, recurre al uso de diversas estrategias de recogida de información como la observación en el aula, las entrevistas en profundidad al profesorado y las entrevistas grupales al alumnado. Los resultados obtenidos ofrecen información acerca de algunas operaciones concretas que el profesorado realiza en aras a simplificar sus materias de enseñanza en formas y recursos asequibles a las estructuras de conocimiento de los estudiantes. Ofrece también información relevante acerca de las características y modo de adquisición del CDC en el contexto universitario.

Palabras clave: Conocimiento Didáctico del Contenido; Conocimiento del Contenido; Aprendizaje Docente; Desarrollo Profesional del Profesorado.

Correspondencia: Trinidad Mentado Labao, Paseo del Valle Hebrón 171, Edificio Levante, Departamento de Didáctica y Organización Educativa, 2º piso, despacho 225, Campus Mundet. E-mail: trini.mentado@ub.edu

* Proyecto de investigación "El conocimiento profesional del profesorado universitario: procesos de construcción y transferencia a la práctica docente" (EDU-2008-05964-C03-01/EDUC) Ministerio de Ciencia e Innovación. Dirección General de Programas y Transferencia de Conocimiento.
\end{abstract}




\begin{abstract}
The objective of this paper is to present the process followed and the results obtained in a research project whose aim was to determine how Pedagogical Content Knowledge (PCK) reveals itself in university education, and specifically in the modifications that teaching staff make in their materials so that students may understand them more easily. The research, carried out through case studies, makes uses of various information gathering strategies, such as classroom observations, in-depth interviews with teaching staff, and group interviews with students. The results obtained offer information about some of the specific adaptations carried out by teachers in order to simplify their teaching materials using forms and resources accessible to students' structures of knowledge. Finally, our results also offer significant information about the characteristics and mode of acquisition of PCK in the university context.

Keywords: Pedagogical Content Knowledge; Content Knowledge; Teacher Learning; Teacher Professional Development.
\end{abstract}

\title{
Introducción
}

En los últimos años, el profesorado universitario se ha visto trabajando en un contexto profesional sujeto a grandes transformaciones. Dejando a un lado los cambios organizativos y de reestructuración de las titulaciones universitarias, la creación del Espacio Europeo de Educación Superior (EEES) ha instaurado en los centros y departamentos una nueva narrativa que insiste en la necesidad de orientar todo el sistema universitario hacia una enseñanza focalizada en el estudiante (Rué, 2007). De este modo, aparecen corrientes pedagógicas que insisten en la importancia del aprendizaje autónomo y colaborativo, del uso de metodologías activas y nuevos métodos de evaluación formativa. Estos cambios, junto a la propuesta de trabajar un conjunto indispensable de competencias específicas y transversales en todas las titulaciones, son elementos que han obligado al profesorado a transformar y adaptar su conocimiento, sus hábitos y creencias, al nuevo escenario educativo. En un momento como el actual, toman especial relevancia aquellos estudios y líneas de reflexión que arrojen información acerca de los contenidos del pensamiento docente y de aquellas formas de enseñanza que obtienen buenos resultados en términos de aprendizajes en el actual EEES.

\section{Propósito del estudio}

Bajo tales premisas, el objetivo de este trabajo es indagar en las manifestaciones del conocimiento profesional del profesorado, y más concretamente del Conocimiento Didáctico del Contenido, en la enseñanza universitaria. En síntesis, las siguientes páginas contienen el relato de un estudio que ofrece información acerca de cómo los profesores universitarios transforman los contenidos de sus asignaturas en formas y recursos pedagógicos eficaces para el aprendizaje de los estudiantes.

\section{Marco teórico}

La investigación contemporánea plantea pocas dudas en torno a algunas cuestiones clave relacionadas con el saber docente. Para muchos autores, el conocimiento del 
profesorado posee un carácter altamente personal y situado al igual que "en el ejercicio de la docencia encontramos distintas formas o enfoques de enseñar" (Tesouro, Corominas, Teixidó y Puiggalí, 2014, p. 171). Diría Eraut (2004) que el saber docente es el recurso cognitivo que los profesores utilizan, de manera personal, en las situaciones concretas de su profesión y que les permite pensar y actuar en ellas. Incluye conocimiento codificado y conocimiento práctico y se traduce en un saber-cómo (know-how) en la forma de habilidades y prácticas, recuerdo de casos y eventos episódicos (Tang, 2010).

Un punto importante de inflexión en el estudio y determinación del saber profesional lo genera la falta de consenso respecto a los elementos que componen su estructura básica (Berry, Loughran, Smith \& Lindsay, 2009; Tardif, 2004). Shulman (1986), pionero en el estudio y delimitación del conocimiento base del profesorado, aludía a siete categorías fundamentales: conocimiento del contenido, conocimiento pedagógico general, conocimiento de los alumnos, conocimiento de los contextos educativos, conocimiento del currículo, conocimiento de los fines educativos y conocimiento didáctico del contenido. Al estudio de Shulman, que pretendía conocer cómo los profesores y profesoras pasaban de la condición de aprendiz a la de docentes y cómo empezaban a construir un saber profesional durante su formación inicial, le siguieron otras muchas investigaciones (Abell, 2008; Berry, Loughran y Van Driel, 2008; Kind, 2009) Pese a la gran variedad de propuestas, en casi todas ellas aparece un constructo con claras implicaciones en la mejora e innovación de la enseñanza universitaria: el Conocimiento Didáctico del Contenido (CDC), un conocimiento específico que se refiere a la forma particular que cada docente tiene de enseñar su especialidad.

Hoy es comúnmente aceptado que los profesores no sólo deben conocer su materia, sino que deben también saber cómo implicar a los estudiantes en un trabajo pedagógico que les ayude a incorporar y construir nociones relacionadas con las materias de enseñanza (Barkley, Cross \& Howell, 2007). En todo ello, el CDC se presenta como un elemento clave.

El CDC incluye la comprensión de lo que significa la enseñanza de un tópico particular, de los principios, técnicas y maneras de representar didácticamente ese contenido y de las concepciones erróneas y dificultades que los estudiantes presentan al aprender (Abell, 2008). Algunas investigaciones recientes sostienen que el CDC tiene su correlato en la enseñanza interactiva, concretamente en la manera en que el docente representa la naturaleza del conocimiento a sus alumnos, en su habilidad para plantear preguntas oportunas en el aula y responder de manera productiva a las intervenciones de los estudiantes (Black, 2008), en el desarrollo y gestión del currículum (Medina y Jarauta, 2013) y en la capacidad de hacer uso de estrategias y recursos (metáforas, analogías, diagramas, explicaciones) que sintonicen con las necesidades de aprendizaje de los estudiantes y con las características internas de las materias de enseñanza (Loewenberg, Hoover \& Phelps, 2008).

En este contexto, toma sentido la investigación que ahora presentamos, pensada para indagar en las manifestaciones del CDC y dirigida a ofrecer información acerca de los procesos de transformación que los profesores realizan para convertir su saber especializado en estructuras pedagógicamente asimilables para los alumnos. 


\section{Metodología y desarrollo de la investigación}

El enfoque adoptado en la investigación responde a una metodología cualitativa, y en concreto a un estudio de casos múltiple. En lo que sigue, ofrecemos algunos apuntes metodológicos y una descripción detallada del desarrollo de la investigación.

\section{Selección de la muestra y trabajo de campo}

En primer lugar, realizamos una encuesta "ad hoc" a 90 profesores experimentados, 11 profesores noveles y 301 alumnos pertenecientes a las facultades de Física, Farmacia, Odontología, Filología, Ciencias Económicas y Empresariales, Enfermería, Derecho y a la Escuela Universitaria de Estudios Empresariales de la Universidad de Barcelona. Los cuestionarios tenían como objetivo localizar a profesores expertos en docencia universitaria. Para ello, se pedía de manera voluntaria a los informantes que aportaran el nombre de cuatro profesores/as considerados buenos docentes, la asignatura que impartían y las razones de la selección. También contamos con la colaboración del Vicerrector de Política Docente y la Unidad Organizativa de Información, Evaluación y Prospectiva (IAP) de la Universidad de Barcelona. La IAP nos facilitó el nombre de 22 profesores que alcanzaban altas puntuaciones en las respectivas escalas de valoración. De todos ellos se seleccionaron los tres que presentaban las más elevadas. Una vez analizada la información, se trianguló para obtener un único listado en el que aparecían los docentes mejor considerados tanto por profesores como por estudiantes.

El primer profesor con el que trabajamos, catedrático de universidad, pertenecía a la Facultad de Física y tenía una experiencia docente superior a los treinta años. La segunda era profesora titular en la Facultad de Enfermería con más de treinta años de experiencia y el tercer profesor era titular en la Facultad de Derecho y contaba con una experiencia docente que excedía los veinte años.

El trabajo de campo se extendió durante los cursos 2009-2010 y 2010-2011 y las principales estrategias de recogida de información fueron la observación no participante, entrevistas en profundidad al profesorado y entrevistas grupales con el alumnado. La siguiente tabla recoge información sobre el trabajo de campo:

Tabla 1

Trabajo de campo. Recogida de Datos

\begin{tabular}{lccc}
\hline & $\begin{array}{c}\text { P1 } \\
\text { Física }\end{array}$ & $\begin{array}{c}\text { P2 } \\
\text { Enfermería }\end{array}$ & $\begin{array}{c}\text { P3 } \\
\text { Derecho }\end{array}$ \\
\hline Observaciones & 18 sesiones, 18 horas & 19 sesiones, 31 horas & 21 sesiones, 32 horas \\
\cline { 2 - 4 } $\begin{array}{l}\text { Entrevistas } \\
\text { profesorado }\end{array}$ & $\begin{array}{c}1 \text { entrevista biográfica } \\
1 \text { entrevista de } \\
\text { análisis práctica }\end{array}$ & $\begin{array}{c}\text { 1 entrevista biográfica } \\
1 \text { entrevista de análisis } \\
\text { práctica }\end{array}$ & $\begin{array}{c}1 \text { entrevista biográfica } \\
1 \text { entrevista de análisis } \\
\text { práctica }\end{array}$ \\
\cline { 2 - 4 } $\begin{array}{l}\text { Entrevistas } \\
\text { alumnado }\end{array}$ & 1 Entrevista grupal & 1 Entrevista grupal & 1 Entrevista grupal \\
\hline
\end{tabular}




\section{Las estrategias de recogida y análisis de información}

La investigación ha seguido un proceso deductivo-inductivo, en el que el trabajo de campo se ha realizado partiendo de ciertos supuestos teóricos previos procedentes de la revisión de la bibliografía. En un primer nivel de análisis, iniciamos las observaciones de aula teniendo en cuenta un conjunto de indicadores extraídos de la revisión de la literatura y de estudios previos realizados por miembros del equipo de investigación (Jarauta \& Medina, 2012; Medina \& Jarauta, 2013). Estos indicadores, como muestra la siguiente tabla, aludían a diferentes formas en que el CDC podía manifestarse en la enseñanza:

Tabla 2

Indicadores deductivos

\begin{tabular}{ll}
\hline Indicadores & Definición \\
\hline Analogías & $\begin{array}{l}\text { Enseñanza de nociones abstractas y poco familiares a través de otras } \\
\text { ya conocidas y accesibles al nivel de comprensión de los estudiantes. }\end{array}$ \\
\hline Conflicto Cognitivo & $\begin{array}{l}\text { Planteamiento de situaciones dilemáticas, de cuestionamiento y con- } \\
\text { flicto a través de situaciones reales o imaginarias que requieran del } \\
\text { análisis y juicio del alumnado. }\end{array}$ \\
\hline Diálogo Reflexivo & $\begin{array}{l}\text { Explicitación del procedimiento con el que los docentes están traba- } \\
\text { jando y las decisiones y motivos que justifican las acciones que realizan } \\
\text { en el aula. }\end{array}$ \\
\hline Ejemplos & $\begin{array}{l}\text { Utilización frecuente de ejemplos para ilustrar o mostrar situaciones, } \\
\text { fenómenos, relaciones u objetos como representativos de aquello que } \\
\text { el docente intenta enseñar. }\end{array}$ \\
\hline Explicitación & $\begin{array}{l}\text { Ejemplificación, en el aula, del modelo cognitivo utilizado por el do- } \\
\text { cente para comprender un contenido concreto de enseñanza. }\end{array}$ \\
\hline Historias Anecdóticas & $\begin{array}{l}\text { Desarrollo de relatos utilizados para reforzar las explicaciones realiza- } \\
\text { das en el aula o para generar un clima que motive a los alumnos hacia } \\
\text { las temáticas de estudio. }\end{array}$ \\
\hline Integración & $\begin{array}{l}\text { Relación que el docente establece entre el contenido de la asignatura y } \\
\text { otras materias afines. }\end{array}$ \\
\hline Disciplinar & $\begin{array}{l}\text { Uso diverso de la interrogación didáctica, en cuanto a sus formas y en } \\
\text { cuanto a los objetivos que persiguen los docentes mediante esta es- } \\
\text { trategia. }\end{array}$ \\
\hline $\begin{array}{l}\text { Interrogación } \\
\text { Didáctica }\end{array}$ & $\begin{array}{l}\text { Estrategias utilizadas por el docente para relacionar el contenido con } \\
\text { aspectos y cuestiones de actualidad y/o con la futura práctica profe- } \\
\text { sional del alumnado. }\end{array}$ \\
\hline
\end{tabular}

Esta propuesta se complementó con otras categorías de carácter inductivo, que emergieron de la recogida de datos realizada en cada una de las realidades concretas del estudio. Son las siguientes: anticipación de contenidos, antropomorfismo, conciencia 
situacional, interpretación didáctica (empatía didáctica), patrones perceptivos, pizarra y construcción de conocimiento, recapitulación de contenidos, reflexión en la acción, reflexión sobre la acción, rigurosidad terminológica, traducción dialógica.

La modalidad de observación adoptada en la recogida de datos fue la no participante y las asignaturas observadas fueron "Fundamentos de mecánica" del grado de física, "Enfermería Médico-Quirúrgica II" de la titulación a extinguir de Diplomado/a en Enfermería y "Derecho Mercantil" del grado de derecho. Las entrevistas se realizaron cuando los datos obtenidos en las observaciones empezaban a ser redundantes (saturación) y apenas aportaban información relevante y novedosa para la investigación. Planificamos dos tipos de encuentros, la entrevista inicial biográfico-profesional y la entrevista de análisis de la práctica. A través de la primera, los profesores pudieron ofrecernos datos relativos a su contexto y a los modos y mecanismos que habían desarrollado para aprender en el aula y fuera de ella. A través de la segunda entrevista, pretendíamos contrastar las interpretaciones que el equipo investigador había realizado de las actividades docentes observadas, con el significado que los profesores y alumnos les atribuían. Para la realización de esta entrevista presentamos a los docentes segmentos de clase, recogidos en formato audiovisual, que parecían aportar elementos de análisis importantes para el objeto de estudio.

Las entrevistas grupales a los estudiantes (entre 4 y 5 estudiantes por cada asignatura observada) fueron realizadas en el momento en el que finalizaba el período de observación con cada uno de los profesores participantes. Las preguntas que se formularon al alumnado respondían a los objetivos de la investigación y a ciertas cuestiones emergentes del análisis preliminar realizado sobre la información obtenida en las observaciones de aula.

Las transcripciones de las entrevistas a profesores y estudiantes y de las observaciones realizadas conformaron el corpus de datos que fue procesado con el programa de análisis de datos cualitativos Atlas-ti con el que desarrollamos los procedimientos analíticos de segmentación, separación, ordenación, búsqueda y recuperación de datos.

Los investigadores del equipo trabajamos inicialmente de manera independiente sobre estos datos. En un segundo momento, contrastamos los análisis individuales para llegar a consensos en las interpretaciones buscando aumentar la credibilidad de los resultados mediante esta triangulación de investigadores (Sandín, 2000).

El método seguido para llevar a cabo el análisis final de datos ha sido el de las comparaciones constantes de Glaser y Strauss (1967). El objetivo final de esta modalidad analítica es la generación inductiva de constructos teóricos, que junto con los núcleos temáticos y las categorías, conforman un entramado conceptual que subsume todos los aspectos de la realidad estudiada y les asigna un sentido y significado nuevos.

Al mismo tiempo que se han ido clasificando los diversos fragmentos del corpus de datos en distintas categorías, éstas han sido comparadas entre sí con el objeto de refinarlas y hacerlas más fiables. De ese modo se ha podido identificar similitudes y diferencias entre los distintos fenómenos estudiados, lo cual ha permitido definir las propiedades básicas de las categorías y una clarificación de sus límites y relaciones lo que, a su vez, ha ayudado a que emerjan metacategorías. 
Por lo tanto, el análisis de los datos procedentes de las observaciones y entrevistas llevado a cabo se dividió en los siguientes niveles progresivos de reducción y estructuración teórica de la información:

NIVEL 1: Segmentación e identificación de unidades de significado y agrupación en categorías descriptivas. En una primera fase, el esfuerzo interpretativo ha estado orientado al desarrollo de descripciones "densas" (Geertz, 1987), es decir, descripciones de los significados que el profesorado y el alumnado utilizan para comprender y dar sentido a los procesos de enseñanza y aprendizaje que han protagonizado. Con ese fin cada investigador realizó la lectura de todas las transcripciones (entrevistas y notas de campo) lo que permitió tener una idea global del contenido de las mismas y conocer los temas nucleares en torno a los cuales se articulaba el discurso de los participantes. En un segundo trabajo de lectura, se llevó a cabo la segmentación del corpus de datos. Para ello, se han considerado unidades de significado aquellos fragmentos que reflejasen una misma idea (fragmentos del texto con sentido semántico). Para esa segmentación o división en unidades de significado se utilizó el criterio del tema abordado y, por tanto, la segmentación del corpus de datos (identificando las unidades de significado) y la codificación de las mismas se han llevado a cabo en una sola operación de modo simultáneo. De ese proceso, emergieron 20 categorías consensuadas por el equipo investigador que cubrían todas las unidades de análisis relevantes para el estudio (346 unidades de significado). Cabe señalar que, a través del método de las comparaciones constantes entre los análisis realizados individualmente, el sistema emergente de categorías fue constantemente refinado, modificado, depurado y redefinido en función de las nuevas unidades que iban apareciendo. A medida que iban apareciendo nuevas unidades de significado se comparaban con las categorías ya existentes para incluirlas en una de ellas; si no era posible, se creaba una nueva categoría que cubriese la idea a la que se refería la nueva unidad de significado. Pero al mismo tiempo, se comprobaban y comparaban las unidades que pertenecían a diferentes categorías ajustando los criterios de pertenencia y asignación lo cual permitió fusionar categorías muy semejantes, dividir aquéllas con un contenido demasiado heterogéneo y redefinir sus etiquetas para que se adecuasen mejor a su contenido. El proceso de construcción del sistema categorial finalizó cuando se empezó a comprobar la saturación de las categorías, es decir, en una nueva lectura individual de las transcripciones no se encontró nueva información que generase la creación de nuevas categorías.

NIVEL 2: Núcleos temáticos emergentes. En un segundo nivel de análisis (y de abstracción) se ha llevado a cabo una agrupación y estructuración de las 20 categorías que han emergido en el primer nivel. Después de un proceso de comparación intercategorías en el que se buscaron similitudes estructurales, teóricas y elementos comunes, han emergido 3 núcleos 
temáticos en torno a los cuales se han agrupado todas las categorías. Estas metacategorías se han construido de manera simultánea e interactiva a la emergencia de las categorías en el primer nivel utilizando para ello los esquemas conceptuales de nuestro marco teórico y de interpretación. Constructos que hemos intentado reformar para captar todas las propiedades de la realidad estudiada tal y como la describían los informantes. Esto quiere decir que las metacategorías o núcleos temáticos que han emergido en este segundo nivel de análisis no son independientes o extraños a las categorías o perspectivas de significado de los profesores y estudiantes participantes. En cierto sentido todas las personas participantes en el estudio están reflejadas en los dominios emergentes que se han definido. Sin duda, la negociación de significados e interpretaciones llevadas a cabo conjuntamente con el profesorado y estudiantes participantes en el estudio ha colaborado en buena medida a tal fin.

Una vez finalizado el proceso, obtuvimos tres grandes metacategorías: (i) la génesis de necesidades de explicación y de expectativas de comprensión en el alumnado, (ii) la dimensión dialógico-reflexiva y (iii) la transformación del saber.

\section{Resultados: La transformación del saber en la enseñanza}

Del conjunto de metacategorías y categorías del estudio, en este artículo presentaremos los resultados que aluden a la metacategoría de la transformación del saber.

De cara a una mayor comprensión de los datos obtenidos, el sistema de notación utilizado para citar las unidades de significado que sustentan las interpretaciones realizadas por el equipo investigador, incluye una referencia numérica mediante la cual identificamos al profesor (P1, P2, P3). Además "E" significa entrevista, "O" Observación y "EA" equivale a fragmentos extraídos de las entrevistas a los alumnos. Asimismo, mostraremos en un recuadro aquellos fragmentos procedentes de las observaciones de clase.

\section{La representación de las realidades científicas a través de ejemplos y analogías}

De entre todos los recursos utilizados en el aula por parte de los tres profesores, el ejemplo y la analogía fueron aquellos que se dieron con mayor frecuencia. La variabilidad en el uso de estos recursos fue tan elevada que pudimos asistir a una multitud de explicaciones en las que los docentes intentaban, a través del ejemplo o analogía, vincular sus materias a los actuales contextos político, económico y social, a situaciones propias de la vida cotidiana y de la práctica profesional.

A propósito de lo comentado, véase en el siguiente episodio de clase cómo la profesora de enfermería utiliza las analogías de la jeringa y el émbolo para hablar de las repercusiones de la presión positiva de la ventilación mecánica en los sistemas respiratorios y cardiocirculatorio:

O9P2: Comencemos por las repercusiones a nivel del sistema respiratorio. [Pasa una diapositiva con una figura de una jeringa y un émbolo que repre- 
senta la entrada de aire (presión positiva) y salida de aire del pulmón.] (...) Aquí tenemos un émbolo que insufla aire a presión al pulmón ¡Es como una jeringa enorme, enorme! Que está insuflando con presión positiva. ¡Esto es un respirador, eh!

O en este otro episodio en el que el profesor de física recurre a ejemplos de la vida cotidiana para explicar el coeficiente de ficción:

O6P1: De hecho ya os dije que estos problemas de la fricción son muy importantes desde el punto de vista tecnológico. Y el gran interés, primero: conseguir que la fricción sea muy grande; es decir, que los adhesivos aguanten mucho (...) Y a la inversa, también se quiere eliminar la fricción. Por ejemplo, las paellas tienen un recubrimiento de un material, que está patentado, que se llama teflón. El teflón es un material polímero que está diseñado justamente para minimizar la fricción.

Como reveló el profesor, este tipo de ejemplos le ayudaban a conectar la física con cuestiones relativas a la experiencia humana, al mundo real y natural. Aproximar la ciencia física a los estudiantes, ayudándoles a pasar de un plano abstracto y conceptual a otro más concreto y cotidiano, era uno de los objetivos que el docente perseguía a través de los ejemplos que utilizaba en el aula:

E1P1: “La idea es conectar la física con el mundo real porque la física debe entenderse como una ciencia natural, como una de las disciplinas que permite entender los fenómenos naturales. La paella y el huevo frito son del mundo real. El tren también es del mundo real, el metro, etc. Y entonces creo que los ejemplos permiten pasar de un plano más abstracto y conceptual a un plano más concreto y cotidiano. Esto a los estudiantes les permite respirar un poco y luego les permite conectar estos dos planos".

Como puede observarse en los anteriores fragmentos, y corroborando las aportaciones de Huertas (2009), los conocimientos académicos y científicos presentan con frecuencia un elevado grado de abstracción. Son conocimientos descontextualizados que necesitan de un anclaje con la realidad para ser entendidos. Por ello, una de las funciones del profesor consiste precisamente en asegurar el anclaje más adecuado entre la capacidad mental del alumnado, sus conocimientos previos y el significado y sentido social y cultural que reflejan y representan los contenidos (Serrano y Pons, 2011). El uso de ejemplos y analogías obligaba a los tres profesores a considerar el punto de partida en que se encontraban los estudiantes al iniciar una explicación y el punto al que deseaban que llegaran al finalizar la sesión o tema. Esto era posible por el amplio conocimiento que los profesores poseían de las características del aprendizaje en la asignatura que impartían. Este conocimiento les permitía interpretar a nivel didáctico la materia y a reconducirla depositándola en recursos y formas de enseñanza cuyo origen era las propias creencias y la experiencia de los estudiantes. 
Como indicó el profesor de física, el conocimiento de los estudiantes procedía de su experiencia en la enseñanza y de su participación reiterada en la asignatura:

E2P1: "Pues me baso, en primer lugar, en mi experiencia previa, en los años que he estado impartido esta asignatura o similar, en diferentes planes de estudio, en más de veinte años de docencia. Esta asignatura la he impartido muchas veces. Y ya sé qué les costará más, qué les resultará más nuevo y qué les resultará más conocido. O sea que tengo mucho conocimiento previo de las dificultades que se encontrará el estudiante".

De las palabras del profesor se infieren dos ideas relevantes acerca del proceso de construcción del CDC. En primer lugar, que el conocimiento de las concepciones que los estudiantes tienen respecto a una idea o concepto puede ayudar a los docentes a planificar acciones que permitan optimizar el proceso de enseñanza-aprendizaje, enriqueciendo a su vez su Conocimiento Didáctico del Contenido (Ruberto, Mac, Calabró \& Rodríguez , 2012). En segundo lugar, es oportuno considerar que el CDC, en su conjunto, se adquiere y perfecciona con la experiencia (Kind, 2009; Rohaan, Taconis \& Jochems, 2010) y se sustenta en el repertorio de construcciones pedagógicas que los docentes desarrollan como resultado de su participación reiterada en procesos de planificación y acción, y de la reflexión sobre la enseñanza de aquellos temas impartidos con mayor regularidad (Hashweh, 2005).

\section{La utilización del saber experiencial y cultural para la enseñanza del contenido científico: el relato de historias anecdóticas en el aula}

De manera paralela, los docentes integraban en sus explicaciones relatos e historias anecdóticas para ilustrar algún concepto abstracto, reducir la complejidad de los fenómenos y tópicos científicos o bien para contribuir a la creación de un clima distendido en el aula.

Un ejemplo, en este caso protagonizado por la profesora de enfermería, se observa en el siguiente fragmento, donde la docente recurre a una anécdota personal para ilustrar el estado de confusión y agitación que manifiestan algunos pacientes de la UCI cuando se les retira la sedación:

O2P2: “El estado mental del paciente puede ser de confusión, de agitación... Puede estar irritable, deprimido. Una vez se retira la sedación, los pacientes presentan un estado mental que no es normal y que cabe la posibilidad o el riesgo de resistencia a la terapia. Y también cabe la posibilidad de que se arranquen todas las vías que llevan y agredan al personal. Yo fui víctima de este tema en una unidad de curas intensivas respiratorias en que trabajé, en que un paciente cada día me iba diciendo que yo era una ladrona y que llamaría a la policía. Y cada día me decía lo mismo. Hasta que un día llegué y la primera cosa que hizo fue levantarse de la cama, arrancárselo todo, no sé de dónde sacó la fuerza aquel enfermo, cogió el palo del suero, 
y empezó a correr detrás de mí, persiguiéndome, diciéndome que yo era una ladrona (alumnos ríen). Estas cosas pueden pasar".

Estas anécdotas, procedentes de su experiencia profesional, contribuían a que los alumnos percibieran los aspectos más relevantes de la práctica de la enfermería de una forma amena, que además fomentaba su comprensión.

Este modo de interpretar el uso de las historias anecdóticas en el aula, coincidía con la percepción que los estudiantes tenían acerca de su eficacia para ilustrar aspectos recónditos de la profesión enfermera:

E1A2: "Yo recuerdo de las primeras clases con ella que a las tres semanas aún recordaba lo que había dado en clase. A las tres semanas estaba en casa y un día salió por la tele algo sobre el ojo y me acordaba calcado de casi toda la clase".

\section{Problematización e interpretación de los contextos de acción para el desarrollo del conocimiento profesional}

Como hemos explicitado en el apartado anterior, algunas de las historias anecdóticas que los profesores explicaban en el aula tenían que ver con el ejercicio de las profesiones para las que formaban. Incluir en el aula evidencias de su experiencia práctica mediante ejemplos y casos anecdóticos, le llevaba a mostrar una parte significativa de aquellos saberes que habían construido por enfrentarse, de manera continuada, a complejas situaciones y a dilemas prácticos de la profesión enfermera. Sin desestimar el abordaje de conocimientos teóricos o conceptuales propios de su materia, la profesora a menudo compartía con los alumnos las convicciones y significados, conocimientos y creencias que habían respaldado algunas de las decisiones y acciones que le habían resultado útiles en la práctica:

E1P2: “También es verdad que de todo lo que explico también lo he vivido en la práctica, salvo contadas patologías que podría decir que no he vivido, pero la mayoría de patologías que cuento y de intervenciones de enfermería sí las he vivido como enfermera. Y, claro, puedo contar anécdotas de lo que me ha pasado, lo que no me ha pasado, lo que he tenido que hacer... Quizás esto también enriquece las clases o las hace más activas o los hace menos aburridas".

Nótese como los anteriores fragmentos denotan por parte de la docente un interés por superar la dicotomía teoría-práctica todavía existente en la enseñanza universitaria, tomando como principio organizador la indagación y reflexión sobre su propia práctica. De este modo, incorporaba de manera intencional referencias que mostraban los conocimientos que los profesionales generan en y desde la práctica, desvelando que su modo de entender y construir el conocimiento era producto de la problematización y continua reinterpretación de los contextos de acción. En suma, hablar de "lo vivido" permitía a la profesora transmitir uno de los rasgos más sobresalientes 
del conocimiento práctico profesional. Rasgo que, en definitiva, los estudiantes debían desarrollar como requisito para su ejercicio profesional: su carácter situado y su naturaleza idiosincrásica.

Por otro lado, la referencia a los contextos profesionales de la abogacía, ayudaba al profesor de derecho a mostrar, en el aula, la aplicación práctica y real de los contenidos trabajados en la asignatura a la realidad actual:

E2P3: “Muchas materias tienen en ocasiones contenidos áridos y difíciles de aprender, pero a menudo dichos contenidos tienen una imbricación con un tema práctico o de actualidad que permite una mejor conexión del estudiante con un tema más abstracto e intangible. Así, los estudiantes entienden mejor el contenido de la asignatura y ven con mayor claridad su aplicación a la realidad vigente".

Por tales motivos, presentaba a los estudiantes casos prácticos que requerían de la comprobación de la teoría en situaciones reales, sujetas al ejercicio de la profesión. El objetivo de formar al estudiante para que "pensara y actuara como un abogado" le llevaba a diseñar situaciones donde la aplicación del conocimiento resultara problemática, donde los estudiantes pudieran percibir las conexiones entre el conocimiento general y los problemas concretos y donde empezaran a construir una identidad vinculada a su futuro ámbito profesional. Práctica que a menudo se complementaba con los comentarios que el profesor hacía respecto a las competencias necesarias para un ejercicio adecuado de la abogacía:

O7P2: "La modificación de la ley del colegio de profesionales, es una las cincuenta y pico leyes que modifica la ley ómnibus. En la práctica uno de los ejercicios importantes es la de navegar por nuestra legislación. Nuestra legislación es desbordante (...) una habilidad que tenemos que desarrollar es leer en vertical identificar donde están los problemas".

A propósito de los resultados anteriores, cabe señalar que los profesores de derecho y enfermería presentaban un elevado compromiso con una de las grandes funciones de la Universidad: formar y preparar para el mundo profesional. Para ello, debían conocer bien el contenido de sus asignaturas pero también saber cómo éste se desarrollaba y manifestaba en los contextos a los que respondía su titulación de referencia. Incorporar en la enseñanza las características de las profesiones requiere del profesorado un cierto conocimiento del contexto profesional, del mundo del empleo y de la evolución y retrocesos de las profesiones. Sin embargo, esta cuestión se presenta a menudo como un elemento conflictivo debido a que el profesorado no siempre posee un saber profesional derivado del ejercicio práctico que vaya más allá de las fronteras de la universidad. Entonces, la adquisición de este tipo de saber requiere de un interés por conocer aquello que sucede en las profesiones, por contactar constantemente con las realidades de los titulados y por incorporar en la enseñanza los últimos avances y conocimientos que surgen de los ámbitos ocupacionales. 


\section{Conclusiones}

El acto de enseñar implica siempre una transformación del saber. En el aula, los profesores actúan como hermeneutas o traductores de saberes. A menudo, convierten el saber científico en saber enseñado y tratan de acompañar a los estudiantes en el transvase que se produce de una representación a otra. Esta reelaboración, que nosotros hemos denominado "transformación del saber", alude a las diversas elecciones que los docentes realizan acerca de cómo encauzar y desarrollar sus explicaciones en el aula. En nuestra investigación esta categoría ha tomado formas diversas pero se ha manifestado fundamentalmente a través de analogías, ejemplos, historias anecdóticas y transferencia a los contextos profesionales. Por lo que pudimos observar, se trataba de creaciones y recursos ideales que los profesores introducían en el aula para provocar la comprensión y la adquisición de los nuevos contenidos de enseñanza. Estas estructuras transportaban el conocimiento que presumiblemente los profesores pretendían que los estudiantes aprendieran.

Además de ello, es oportuno señalar otras conclusiones referidas al origen y desarrollo del CDC. En primer lugar, es estructuralmente un conocimiento práctico en el que el marco disciplinar bajo el que actúan los profesores y el nivel de comprensión que poseen de éste afecta a la calidad de esa transformación. Se adquiere y perfecciona con la experiencia y requiere de la confrontación reflexionada entre las estructuras disciplinares y el conocimiento de las situaciones de enseñanza y de las características que adopta el aprendizaje del alumnado. De carácter integrador, el CDC implica un tipo de razonamiento que permite a los profesores reorganizar y transformar todos los saberes profesionales de los que disponen para la enseñanza de sus materias. En nuestro estudio pudimos observar que los tres profesores ideaban ejemplos, analogías y anécdotas que partían de una gran variedad de saberes: conocimiento del contenido, del alumnado, del contexto profesional, de la historia de las disciplinas. También relacionaban los contenidos de sus asignaturas con situaciones de la práctica profesional para la que formaban. Esta acción era una clara manifestación del conocimiento que los tres profesores poseían acerca del contexto profesional al que contribuía la titulación en la que ejercían su función docente.

Y finalmente, el CDC es un tipo de conocimiento que a medida que se va desarrollando permite al docente generar más y mejores transformaciones y procesos de razonamiento didáctico. Por su relación con todas las categorías del conocimiento profesional, el CDC será, a su vez, cada vez más elaborado a medida que los docentes adquieran una mayor comprensión del contenido y de los contextos de enseñanza, del conocimiento pedagógico y de los alumnos, y a medida que se enfrenten a situaciones en las que deban utilizar su CDC para analizar y revisar sus prácticas docentes.

\section{Bibliografía}

Abell, S.K. (2008). Twenty years later: Does pedagogical content knowledge remain a useful idea? International Journal of Science Education, 30(10), 1405-1416.

Barkley, E., Cross, P. \& Howell, C. (2007). Técnicas de aprendizaje colaborativo. Madrid: Morata. 
Berry, A., Loughran, J., Smith, K. \& Lindsay, S. (2009). Capturing and enhancing Science Teachers 'Professional Knowledge. Res Sci Educ, 39, 575-594.

Berry, A.; Lougrhan, J. y Van Driel, J.H. (2008). Revisiting the roots of pedagogical content knowledge. International Journal of Science Education, 30(10), 1271-1279.

Black, J.W. (2008). Content Knowledge and Pedagogical Content Knowledge of Algebra Teachers and Changes in Both Types of Knowledge as a Result of Professional Development. En M. Quazi (Ed.), Proceedings of the $5^{\text {th }}$ Annual TEAM-Math Partners hip Conference Pre-Session (pp. 30-40). Tuskegee, AL: Tuskegee University

Eraut, M. (2004). Informal learning in the workplace. Studies in Continuing Education, 26(2), 247-273.

Geertz, C. (1987). La interpretación de las culturas. Barcelona: Gedisa.

Glaser, B. \& Strauss, A. (1967). The discovery of Grounded Theory. Chicago: Aldine.

Hashweh, M.Z. (2005). Teacher pedagogical constructions: A reconfiguration of pedagogical content knowledge. Teachers and Teaching: Theory and Practice, 11(3), 273-292.

Huertas, J.A. (2009). Aprender a fijarse metas: Nuevos estilos motivacionales, en Pozo, J.I. \& Pérez Echeverría, M.P. (coord.) Psicología del aprendizaje universitario: la formación en competencias, (pp.164-181). Madrid: Narcea.

Jarauta, B. \& Medina Moya, J.L. (2012). Fuentes y procesos de aprendizaje docente en el contexto universitario. Revista Española de Pedagogía, 252, 337-353.

Kind, V. (2009). Pedagogical content knowledge in science education: Potential and perspectives for progress. Studies in Science Education, 45(2), 169-204.

Loewenberg, D., Hoover, M. \& Phelps, G. (2008). Content Knowledge for Teaching. What Makes it Special? Journal of Teacher Education, 59(5), 398-407.

Medina, J.L. \& Jarauta, B. (2013). Análisis del Conocimiento Didáctico del Contenido de tres profesores universitarios. Revista de Educación, 360, 600-623 DOI: 10.4438/1988592X-RE-2011-360-131

Rohaan, E., Taconis, R. \& Jochems, W. (2010). Analysing teacher knowledge for technology education in primary schools. International Journal of Technology and Design Education, 22(3), 271-280.

Ruberto, L.A.M.; Mac, W.P., Calabró, A. \& Rodríguez, J. (2012). Conocimiento Didáctico del Contenido (CDC) en la enseñanza universitaria de Biotecnología. El caso de la velocidad específica de crecimiento microbiano $(\mu)$. Revista Eureka sobre Enseñanza y Divulgación de las Ciencias, 9 (3), 353-360.

Rué, J. (2007). Enseñar en la universidad. El EEES como reto para la Educación Superior. Madrid: Narcea.

Sandin, M. P. (2000). Criterios de validez en la investigación cualitativa: de la objetividad a la solidaridad. Revista de Investigación Educativa, 18(1), 223-242.

Serrano, J.M. y Pons, R. (2011). El constructivismo hoy: enfoques constructivistas en educación. Revista Electrónica de Investigación Educativa, 13(1), 1-27.

Shulman, L.S. (1986). Those who understand: knowledge growth in teaching. Educational Researcher, 15(2), 4-14.

Tang, S.Y.F. (2010). Teachers 'professional knowledge construction in Assessment for Learning. Teachers and Teaching: theory and practice, 16(6), 665-678.

Tardif, M. (2004). Los saberes del docente y su desarrollo profesional. Madrid: Narcea. 
Tesouro, M., Corominas, E., Teixidó, J. \& Puiggalí, J. (2014). La autoeficacia docente e investigadora del profesorado universitario: relación con su estilo docente e influencia en sus concepciones sobre el nexo docencia-investigación. Revista de Investigación Educativa, 32 (1), 169-186. doi: http://dx.doi.org/10.6018/rie.32.1.172771

Fecha de recepción: 27 de febrero de 2015.

Fecha de revisión: 27 de febrero de 2015.

Fecha de aceptación: 29 de marzo de 2016. 
